\title{
Logique Communicationnelle, Posture et Division dans les Familles Élargies: Cas de Baazra, en Côte d'Ivoire
}

\author{
Gala Bi Tizié Emmanuel, \\ Enseignant-chercheur à l'Institut d'Ethno-Sociologie, \\ UFHB Abidjan, Côte d'Ivoire \\ Bini Koffi Roland, \\ Chercheur à l'Institut d'Ethno-Sociologie, \\ UFHB Abidjan, Côte d'Ivoire
}

Doi:10.19044/esj.2020.v16n16p172 URL:http://dx.doi.org/10.19044/esj.2020.v16n16p172

Résumé

Cet article vise à comprendre la dynamique communicationnelle à Baazra, village de la commune de Zuénoula, au centre-ouest de la Côte d'Ivoire, ses causes et conséquences. Il s'agit d'une étude qualitative qui s'appuie sur une méthode d'échantillonnage non probabiliste dite par choix raisonné, et menée à partir d'entretiens semi-dirigés avec une vingtaine d'individus. En matière de résultats, l'étude a mis en exergue la position sociale comme facteur déterminant dans la prise de parole, le droit à la parole étant légitimé par des facteurs socio-économiques. Aussi, la propagande médiatique du modèle démocratique occidental a contribué à modifier les logiques communicationnelles à Baazra. Toutes ces mutations sont la base des conflits communautaires; d'où le recours à la médiation des pouvoirs publics qui est souvent contesté.

Mots clés : Logiques communicationnelles, posture, intervenant, famille, développement 


\title{
Communicational Logic, Posture, and Division within Extended Families: The Case of Baazra in Côte d'Ivoire
}

\author{
Gala Bi Tizié Emmanuel, \\ Enseignant-chercheur à l'Institut d'Ethno-Sociologie, \\ UFHB Abidjan, Côte d'Ivoire \\ Bini Koffi Roland, \\ Chercheur à l'Institut d'Ethno-Sociologie, \\ UFHB Abidjan, Côte d'Ivoire
}

\begin{abstract}
This paper focuses on understanding the communicational dynamics, its causes, and its consequences in Baazra-a village located at the municipality of Zuenoula in the mid-west of Côte d'Ivoire. This qualitative study is based on a non-probability sampling approach which is known as a purposive sampling. It was carried out through semi-structured interviews administered to twenty respondents. The results of this survey show that the social position is a determining factor in speaking. In other words, the right to speak is inherent to socioeconomic factors. Moreover, the media propaganda of the western democratic model has contributed in changing the communicational logics in Baazra. All these changes forms the basis for community conflicts; hence this has resorted to the mediation of State authorities which is often contested.
\end{abstract}

Keywords: Communicational logic, posture, speaker, family, development

\section{Introduction}

\section{I.1 Contexte}

La communication sous toutes ses formes, vise à établir et à entretenir une relation sociale, à partir d'échanges. Communiquer est un processus régi par des normes spécifiques selon les milieux sociaux. Chaque société, selon sa culture, a une manière particulière de communiquer. Qu'elle soit directe ou indirecte, la communication en Afrique est une «arme » qui, utilisée dans les normes prescrites peut atteindre des résultats efficients. En d'autres termes, la communication dans les milieux organisés d'Afrique influence la qualité de la relation. Communiquer est un art appris en communauté dès que commence le processus de socialisation. C'est dans cette logique que Kwabenan Ngboko (2005) reprend le proverbe Bron suivant : « kassabiyakassa, kassakassaYè Ya, 
kassakassa a kassakrongron », qui signifie : «toute parole est parole, parler est facile et difficile. Qui veut parler doit parler clair, bien, vrai». Cette idée traduit la valeur de la communication dans la culture africaine en général et ivoirienne en particulier ; car c'est d'elle que dépend la consolidation des rapports sociaux. En un mot, la gestion de la relation communicationnelle traduit le type de relation qui existe entre deux ou plusieurs individus.

Dans le registre ivoirien où la communication se traduit, entre autres, par la parole, le ton, la gestuelle, le moment de prise de parole et le temps de parole, le poids des mots détermine la hiérarchie, l'humilité et l'entente entre les différents membres d'une famille ou d'une communauté. Mais, la communication sociale ou participative n'étant toujours pas facile, c'est le lieu d'indiquer que cette étude se situe dans un contexte marqué de certaines contradictions dont les plus saillantes sont la scission du villlage de Baazra en deux entités et des conflits de chefferie.

\section{I.2. Problématique}

La prise de parole est aussi naturelle que complexe. Cela est encore plus perceptible lorsque la famille est élargie, car tout ce que l'on dit peut faire l'objet d'interprétation d'une manière ou d'une autre. Dans ce contexte, les ressortissants d'un village, se revendiquant de la même famille, s'organisent autour de la tradition et des aînés qui ont un rôle primordial dans la gestion des relations. Ainsi, l'organisation villageoise se reproduit avec, en substance, une mesure dans la communication, facteur de régulation de la relation familiale.

Cependant, la dynamique sociale et le processus d'acculturation ont contribué à modifier progressivement les habitudes de communication au sein des familles élargies en Côte d'Ivoire. La culture occidentale a modifié les rapports et la structure de la communication entre membres d'une communauté villageoise. Ainsi, certains villages rencontrent des difficultés dans leur mode de fonctionnement actuel. De simples échanges d'informations peuvent être sources de conflits. Il se pose donc de plus en plus un problème communicationnel qui engage l'intervenant et l'intervention, aussi bien entre différents groupes sociaux, qu'entre membres d'un même groupe.

La zone rurale de Baazra dans la commune de Zuénoula, au centreouest de la Côte d'Ivoire n'échappe pas aux conflits liés à la dynamique communicationnelle. En effet, les contradictions d'idées entre ressortissants de Baazra sur les questions de politique villageoise voire du développement aboutissement le plus souvent à des écarts de langages et même à la rupture communicationnelle. Ces problèmes de communication sont une des causes profondes de la scission dudit village en deux parties (Baazra 1et 2) et des problèmes de chefferie récurrents. En d'autres termes, les mutations liées aux modèles communicationnels constituent une problématique essentielle à 
Baazra. Les efforts de résolution de ces conflits par des ressortissants dudit village résidant à Abidjan (capitale économique de la Côte d'Ivoire) et réunis au sein d'une mutuelle de développement, peinent à aboutir, du fait de la complexité des divisions liées à divers enjeux.

Ces constats nous amènent à nous poser les questions suivantes : quels sont les changements liés au processus communicationnel à Baazra ? Quels sont les facteurs à la base de cette dynamique communicationnelle ? Quelles sont les conséquences et suggestions qui se dégagent des conflits nés de ladite dynamique communicationnelle ?

\section{I.3. Objectifs}

Comme objectif général, cette étude vise à comprendre la dynamique communicationnelle à Baazra, ses causes et conséquences.

De façon spécifique, il s'est agi de :

- décrire les changements entre la communication traditionnelle et la communication moderne à Baazra;

- identifier les facteurs liés à ces changements ;

- relever les conséquences relatives à cette dynamique communicationnelle.

\section{Approche Methodologique \\ II.1. Champ géographique et échantillon}

Baazra est une zone rurale de la commune de Zuénoula, au centreouest de la Côte d'Ivoire. Il est scindé en deux (2) villages à savoir, Baazra 1 et Baazra 2. La présente étude concerne les ressortissants de ces deux (2) localités d'ethnie gouro (issue des mandés du sud). Le recensement général de la population ivoirienne de 2014 a estimé la population des deux villages à 1113 habitants.

Cette étude est de type qualitatif. Elle prend appui sur la technique d'échantillonnage à choix raisonné pour interroger vingt (20) personnes ressources. Le choix de ladite technique est justifié par la complexité des conflits de chefferie. Le sujet traité requiert la connaissance de l'histoire du village et la participation des enquêtés à la gestion des affaires villageoises. L'échantillon se doit donc d'être représentatif et susceptible de fournir des informations précises. C'est pourquoi l'enquête a concerné les acteurs suivants :

- Les autorités villageoises (02 chefs), en qualité de premiers gestionnaires de ces localités ;

- les représentantes des femmes (05), elles participent régulièrement aux rencontres villageoises et prennent la parole au nom des femmes ;

- les représentants des jeunes (03), ils parlent au nom de la jeunesse et participent à ce titre à toutes les rencontres; 
- des chefs de familles de la communauté villageoise (05), ils symbolisent les conseillers de la chefferie et portent la voix de leurs familles respectives ;

- les représentants de la Mutuelle de Développement de Bazra-Klaazra (05), ils sont impliqués dans toutes des activités de développement communautaire et de médiation.

\section{II.2. Technique, outils de collecte de données et méthode d'analyse}

La technique pour la collecte des données auprès des enquêtés est l'entretien semi-dirigé, avec comme instrument de collecte un guide d'entretien, structuré comme suit :

- identification de l'enquêté ;

- changements liés à la dynamique communicationnelle en termes d'organisation, d'intervenant, de posture et d'intervention ;

- facteurs explicatifs des changements induits par la dynamique communicationnelle ;

- conséquences et suggestions.

La méthode d'analyse dialectique a servi à comprendre la dynamique communicationnelle à Baazra et à en rendre compte.

\section{Resultats et Discussion}

\section{III.1. Résultats}

\section{III.1.1. Changements liés à la dynamique communicationnelle à Baazra} III.1.1.1. Fondements historiques (structure, intervenant, posture, intervention)

Pour Lohisse (1999), la communication, d'un point de vue socio anthropologique, s'appuie sur un ensemble de règles, de rites, de normes socioculturelles. A l'origine, Baazra était un seul village et disposait d'une unique organisation communicationnelle et politique (de type vertical), animée par la chefferie et les chefs de quartiers. Cependant, du fait d'une divergence d'idéologies politiques (d'une part les partisans du Parti Démocratique de Côte d'Ivoire et les progressistes de l'autre), Baazra a fait l'objet d'une scission en deux entités (Baazra 1 et 2), sans pour autant remettre en cause l'organisation globale de base. Chaque entité ainsi constituée, comprend des quartiers composés de clans ou de grandes familles.

Baazra 1 comprend quatre clans pour quatre quartiers comme l'indique le tableau ci-après. 
Tableau 1. Répartition des habitants de Baazra 1 par clans, quartiers et leurs significations anthropologiques

$\begin{array}{llll}\text { Clans } & \text { Signification } & \text { Quartiers } & \text { Signification } \\ \text { Drènrin } & \text { Descendance de Drè } & \text { Drè-fla } & \text { Village de Drè } \\ \text { Siinrin } & \text { Descendance de Sii } & \text { Sii-zra } & \text { Héritage de Sii } \\ \text { Zoronrin } & \text { Descendance de Zoro } & \text { Zoro-fla } & \text { Village de Zoro } \\ \text { Baanrin } & \text { Descendance de Baa } & \text { Baa-zra } & \text { Héritage de Baa }\end{array}$

Il ressort de ce tableau que Baanrin (constitué des descendants de Baa) est la famille souche du village de Baazra (héritage de Baa en ethnie gouro).

Baazra 2, quant à lui, compte trois (03) clans pour trois (03) quartiers (voir le tableau ci-après).

Tableau 2. Répartition des habitants de Baazra 2 par clans, quartiers et leurs significations

Clans

Drènrin

Siinrin

Signification

Descendance de Drè

Goulinrin
Descendance de Sii

Descendant de Gouli
Quartiers

Drè-fla

Sii-zra

Baligui
Signification

Village de Dré

Héritage de Sii

dans le bambou

Les Tableaux 1 et 2 indiquent que les clans : Drènrin et Siinrin sont autant représentés sur le territoire de Baazra 1 que sur celui de Baazra 2. Ces dénominations communes aux clans de Baazra 1 et 2 constituent donc du point de vue anthropologique un indicateur d'appartenance à un même groupe culturel.

Les chefs de villages confirment cette information en ces termes :

- «les Drènrin et les Siinrin sont les clans de base de Baazra » ( chef de Baazra 1);

- "le village a été fondé par les Drènrin et les Siinrin » (chef de Baazra 2).

Il ressort aussi de l'enquête que le père fondateur de Baazra 2 appartient au clan « Goulinrin ».

Par ailleurs, à Baazra 1 ou 2, la prise de parole en public, selon les principes traditionnels, respecte la hiérarchie. En la matière, un privilège est réservé au chef du village et à ses notables. Il en est de même des chefs de quartiers ou de grandes familles. Ainsi, la communication obéit-elle à un ordre clair qui respecte l'organisation villageoise.

Cette disposition est aussi bien reconnue par la chefferie que par les jeunes et les autres membres de la communauté :

"Par le passé, tous n'avaient pas le droit de prendre la parole publiquement $»$ (chef du village de Baazra 1).

"Au plan traditionnel, ce sont les chefs de familles qui ont droit à la prise de parole. Ils représentent les doyens. Les plus jeunes écoutent leurs ainés » (chef du village de Baazra 2). 
"Quand on doit régler une affaire au village, le chef du village délègue une partie de son pouvoir aux chefs defamilles (les patriarches) pour en parler. Au terme de l'instruction de l'affaire, ces patriarches retournent la parole au chef du village qui donne le verdict » (président des jeunes).

«Avant le respect des ainés était de mise. Il y avait un respect total des institutions villageoises et des aînés. Les gens ne parlaient pas n'importe comment » (membre de la mutuelle de développement des ressortissants de Baazra).

La question du respect de la primogéniture revient constamment au cours des échanges avec les enquêtés. La communication en public est liée au droit d'aînesse et à la position sociale de l'individu dans l'organisation politique. Le porte-parole du clan est une personne âgée et consensuelle. Une fois désigné, ce dernier a le droit mais aussi le devoir de s'exprimer au nom de tous. Les discussions se déroulent généralement entre les chefs de quartiers et le chef principal qui prononce le mot de fin.

\section{III.1.1.2. Fondements actuels (structure, intervenant, posture, intervention)}

Bien que rappelées à l'ordre par des patriarches sur l'importance du respect de la tradition et des normes coutumières, les communautés villageoises de Baazra 1 et 2 ont adopté de nombreux changements en matière de prise de parole. C'est ce que le chef du village de Baazra 1 affirme dans les propos suivants :

«Lors de nos délibérations, les patriarches nous disent souvent que notre manière de faire est incompatible avec l'époque passée. En réalité, ils n'apprécient pas nos manières de faire, parce que les nouvelles générations agissent d'égal à égal avec leurs aînés ou tentent de les supplanter. Ce que les aînés interdisent, c'est ce qui tente les jeunes ».

En réalité, la prise de parole en public à Baazra, à l'heure actuelle, est caractérisée par la liberté d'expression. A cet effet, le chef de Baazra 1 continue et témoigne :

"L’époque ancienne et l'époque nouvelle sont incomparables. Maintenant, tous s'expriment librement ».

Cette liberté d'expression est liée au système politique actuellement en application en Côte d'Ivoire. De fait, en partant du principe de la démocratie, l'organisation administrative du pays a influencé l'organisation villageoise à Baazra. L'autorité de la chefferie villageoise est légitimée par l'administration civile. Les chefs sont désormais nommés, par arrêté préfectoral, puis depuis mars 2019 par la Chambre Nationale des Rois et Chefs Traditionnels de Côte d'Ivoire (CNRCT), en application de la loi n ${ }^{\circ} 428$ du 14 juillet 2014.

Il revient donc au chef de village de valoriser la culture, de faire en sorte que les us et coutumes soient respectés et d'assumer son rôle 
d'administrateur. Pour les ressortissants de Baazra, les chefs sont un peu plus préoccupés par l'administration que par le bien-être de leurs administrés et de la coutume. En conséquence, le mode de communication se modernise progressivement et laisse place à cette liberté d'expression qui signifie ici « ouverture de la parole à tous » (d'après un membre de la communauté villageoise de Baazra I).

La prise de parole est donc légitimée par l'arrêté de nomination administrative. Cela est traduit par le verbatim suivant :

«Le droit de parler du chef est déterminé par l'arrêté de nomination préfectoral dont il a copie » (président des jeunes de Baazra I).

Un autre symbole de la communication actuelle, c'est la cohabitation de l'oralité et de l'écrit (avec la prédominance du second).

"Le grand changement que le village a connu en termes de communication, c'est qu'aujourd'hui tout est mis sur papier. Ce n'est plus uniquement par l'oralité qu'on s'exprime. On le fait également par écrit. Les règlements sont écrits, ce n'est pas par voie orale » (un jeune du village).

D'après un chef de quartier de Baazra 2 : «aujourd'hui, l'oralité a fait place aux documents. Seuls les documents officiels comptent ».

«Désormais, on est obligé de prendre notes et de faire des procèsverbaux après les réunions. Vous comprenez comment les jeunes ont eu leur place » (un chef de quartier à Baazra 2).

La société traditionnelle a toujours privilégié l'oralité. Les mutations sociales induites par l'adoption de nouveaux modes de légitimation des chefs ont un impact direct sur les symboles et le processus de mémorisation des faits importants dans la zone rurale de Baazra.

\section{III.1.2. Facteurs de la dynamique communicationnelle III.1.2.1. Développement socioéconomique}

Si la dynamique sociale semble naturelle, il n'en demeure pas moins que les acteurs sociaux, continuellement en concurrence, accélèrent la cadence. En comparaison à des villages voisins comme celui de Beiziaka (à environ 5 kilomètres), où le développement communautaire est plus observable à travers un plan de lotissement moderne, l'électrification du village, l'adduction d'eau potable, des infrastructures scolaires et de santé, les ressortissants de Baazra font des efforts d'entreprendre des actions de développement. Ils y travaillent quotidiennement, malgré des difficultés telles que celles évoquées par une représentante des femmes de Baazra 2 :

"Chez nous c'est difficile, Baazra n'arrive pas à se développer. Quand survint une situation difficile, tant que le président des jeunes n'intervient pas, rien ne va de l'avant. Le chef $d u$ village n'arrive pas à promouvoir le développement. Quand des difficultés surviennent, nous 
sommes obligés de recourir à nos parents qui sont à Abidjan afin de nous aider à les résoudre ».

Dans ce contexte, les populations de Baazra sont amenées à diluer leur culture communicationnelle. De plus en plus, ils permettent à tous de s'exprimer, non pas parce que cela est recommandé par la tradition, mais parce que cela pourrait servir au développement économique et social du village. La posture moderne est de plus en plus privilégiée.

"Il faut aussi s'appuyer sur les forces vives du village, c'est-à-dire les cadres et la mutuelle. Ceux-ci, en fonction de leurs positions sociales, ont le plus souvent une perception plus large des choses que leurs parents du village. Du fait de leur présence en ville et de leur niveau d'étude élevé, ils jouissent de certaines expériences que ceux du village. Ces expériences, ils peuvent les mettre à profit, pour le développement du village »(chef de Baazra 1).

«Nous en appelons à tous les cadres originaires de Baazra, où qu'ils soient, afin qu'ils viennent nous aider à faire avancer les choses » (chef de Baazra 2).

Dans ces propos, on remarque que la position sociale de l'intervenant est une caractéristique déterminante. En d'autres termes, parler est une chose, mais être écouté en est une autre. Si la liberté d'expression est de mise aujourd'hui, il n'en demeure pas moins que la posture de l'intervenant valide ou non son intervention. Dans ce contexte, l'éducation scolaire est un déterminant et un cadre de modification des normes, du fait de la diversité du public scolaire et de l'intégration de nouvelles cultures que lesdits cadres partagent au village (Abdallah-Pretceille \& Porcher, 1996).

En matière de développement, il faut également tenir compte de la pression de la jeunesse. Ce facteur est déterminant parce qu'il traduit la capacité à se défendre et à influencer des décisions. En effet, il s'agit ici de la révolte des jeunes et de leurs revendications. Avec leur fougue et leur importance démographique, les jeunes s'imposent aux personnes âgées.

"Aujourd'hui, ce sont les jeunes qui ont la parole », témoignait un autre représentant de la jeunesse.

L'on assiste à un conflit intergénérationnel qui se traduit par la désobéissance et la non reconnaissance de la hiérarchie en matière de communication.

A cet effet, un responsable de jeunes affirme : «il y a des fois où les jeunes sont obligés de prendre leurs responsabilités ».

"Il est clair que les jeunes sont souvent trop pressés de prendre la parole et finalement ils disent des bêtises. Avant, nous pensions d'abord à la cohésion sociale. Maintenant, c'est la position sociale et l'argent qui compte », nous confiait un chef de famille, membre de la notabilité.

Cette dernière déclaration pointe du doigt les conflits intergénérationnels et évoque également la recherche du profit personnel. 
L'individualisme est une autre composante du développement économique et social qui a impacté la dynamique communicationnelle problématique.

"La course à l'argent fait que les gens n'ont plus d'honneur. C'est l'intérêt personnel qui prime» (chef de famille à Baazra 2).

Un membre de la mutuelle de développement des ressortissants de Baazra ajoute ce qui suit : "aujourd'hui, certains intervenants dans les villages gouro cherchent à dominer les autres. Chacun veut montrer qu'il est plus fort, qu'il est propriétaire terrien ».

"Certains recherchent le développement du village, d'autres par contre visent la renommée et l'intérêt financier, surtout que la tendance actuelle consiste à payer les chefs. Par exemple, à Bö̈zra, un chef a été installé par le préfet de Zuénoula, alors que le précédent chef voulait se maintenir au pouvoir» (un membre de mutuelle en focus group).

La course à l'argent détermine donc les stratégies des acteurs à se maintenir au pouvoir. En effet, l'administration ivoirienne a fait aujourd'hui des chefs de villages des salariés. Cette nouvelle donne a rendu les transitions politiques plus complexes, les sortants essayent de se maintenir, tandis que les rentrants s'empressent de s'installer. Les conflits d'intérêts sont donc récurrents à Baazra.

Avec le temps, la course à l'argent et au positionnement ainsi que le développement économique et social basé sur des valeurs démocratiques ont bouleversé les fondements culturels, communicationnels et modifié les relations sociales.

\section{III.1.2.2. Propagande médiatique et problématique de la communication}

L'enquête a révélé que les médias occupent une place importante dans le processus d'adoption de nouvelles formes de communication des ressortissants de Baazra.

Au plan culturel, les médias influencent les relations entre les jeunes et les aînés. Pour les jeunes, ce changement est plutôt un point positif. C'est du moins ce que soutient le président des jeunes de Baazra :

"Aujourd'hui, la communication se passe bien, puisque nous avons laissé beaucoup de choses d'avant et trichons maintenant sur les blancs ».

Les autorités villageoises aussi reconnaissent que les médias ont favorisé la liberté d'expression. A cet effet, un chef de famille de Baazra 1 note :

"Actuellement, avec l'arrivée des blancs, chacun est libre de prendre la parole. Si non avant, tant qu'on ne t'a pas donné la parole, tu n'as pas le droit de parler publiquement, devant ton aîné ». 
Cela se traduit par la facilité des jeunes à s'exprimer face aux personnes âgées. Ces derniers reconnaissent qu'ils ont subi l'influence du modernisme et des Technologies de l'Information et de la Communication.

L'autre apport incontournable des médias à la dynamique communicationnelle réside dans l'accès à l'information. L'essor des réseaux sociaux numériques bouleverse et transforme profondément les pratiques médiatiques et la gestion de l'information (Kadjabika et al., 2018). A propos, un ancien président de la mutuelle de développement à Baazra a affirmé ce qui suit :

«Avant, les informations, on les recevait seulement de la chefferie, de la notabilité du village. Le chef pouvait décider de l'information à donner ou pas. Mais aujourd'hui, avec les médias, les TIC, on voit tout ce qui se passe à Abidjan, ce qui se passe même en dehors de nos frontières, on est informé. Tout ça a une influence sur la communication dans le village. Aujourd'hui, avec le téléphone, on peut communiquer avec les parents au village à tout moment, idem avec les réseaux sociaux. Tous ces moyens ont changé les modes de communications dans nos villages. Avant, on utilisait le tam-tam pour communiquer selon les évènements. La manière de jouer cet instrument variait selon la nature de l'évènement (joie, paix). Maintenant, on n'a plus besoin de toutes ces choses. Le téléphone suffit pour que l'information circule, sans qu'elle soit gérée par le chef du village ou des personnes spécifiques ».

La communication a été orientée pendant longtemps à Baazra. L'intervenant était connu, tout comme sa posture. L'intervention était maitrisée et le message dirigé vers l'objectif de préservation de la cohésion sociale. Dans ce contexte, les intervenants étaient crédibles, respectés et incontournables. Les médias ont modifié les rapports à la communication. En prenant la parole, celui qui s'exprime se doit d'être plus créatif pour capter l'attention de tous.

Les médias ont aussi influencé l'éducation et les principes de communication entre les membres de la famille ou de la communauté.

"Ces médias ont apporté des mutations au niveau de l'éducation des jeunes du village. Ce sont eux qui sont les plus familiarisés à ces outils de communication. Ils en ont une connaissance plus nette que leurs ainés » (un chef de famille de Baazra 2).

Les médias font la promotion d'un type de rapport '"enfant-parent' ou "jeune-adulte" qui ne rentre pas dans les principes traditionnels gouro. A Baazra, pour rappel, seul le chef de famille avait la parole et parlait au nom de tous. Les plus jeunes se devaient de passer toujours par lui. Dans la culture occidentale dont la promotion est faite dans les médias, la communication est directe. Les TIC modifient ostensiblement les relations sociales, les rapports de pouvoir, l'action et les médiations rendant «possible » un nouvel ordre social, politique et démocratique (Kajabika \& Kiyindou, 2018). Les enfants 
ou les jeunes ont le choix et s'adressent directement à leurs parents ou à leurs aînés. Les plus jeunes prennent des initiatives sans en informer leurs aînés. C'est ce que traduit cette pensée du président de la mutuelle de développement de Baazra :

«Le plus jeune peut organiser une cérémonie de dot chez lui sans en informer son aîné, alors que par le passé, peu importe vos rapports, tout s'organise chez le patriarche ».

Les médias ont favorisé une acculturation et permis de passer d'une communication indirecte à une communication directe, c'est-à-dire, d'une communication qui passait impérativement par les aînés à une communication ouverte, sans porte-parole, ni protocole.

\section{III.1.3. Conséquences}

Une communication mal maitrisée est facteur de conflit fratricide (CDVR, 2014). La problématique liée à la dynamique communicationnelle à Baazra a de nombreuses conséquences sur la vie communautaire et surtout sur les rapports sociaux.

"Les problèmes de chefferie que Baazra 2 a connus s'inscrivent dans ce registre. Pendant plus de dix ans, il n'y avait pas de chefferie véritable dans ce village. Le chef d'alors a été remplacé par un intérimaire. La mutuelle du village s'est essayée à résoudre cette équation en approchant les acteurs dudit village afin de comprendre ce problème de chefferie et contribuer à sa résolution. Dans le processus de résolution de la crise, la MUDEBAK a convoqué une réunion à laquelle ledit chef de village a confirmé qu'il y prendrait part. Mais au jour de la rencontre, il n'a pas honoré de sa présence cette réunion. Après avoir instruit le dossier, ses anciens notables et les villageois ont trouvé bon de confier ce poste de responsabilité à une de nos sours, qui est d'ailleurs fille du fondateur de Baazra 2. C'était une grande première dans la région gouro (c'était une proposition). Puisque cette proposition est venue des villageois eux même, la MUDEBAK a accédé à leur requête».

Cette déclaration du président de la mutuelle de développement résume certaines conséquences nées des mutations au niveau communicationnel.

La première conséquence des modifications dans le mode de communication, c'est la récurrence des conflits liés à la chefferie. Depuis quelques années, Baazra 2 est le lieu d'un conflit ouvert entre deux (02) groupes de personnes : les partisans du chef sortant (qui refuse de céder son fauteuil) et ceux du chef entrant. En d'autres termes, l'application du système administratif moderne à Baazra 2 suscite de nombreux problèmes. Car, dans ce village, le chef qui a été nommé par arrêté préfectoral de Zuénoula, est remis en cause au détriment d'un autre, reconnu par les villageois. 
La seconde conséquence est la division des familles, les sentiments de frustration qui débouchent sur les atteintes mystiques et la non-participation de certains aux réunions de famille et de mutuelle.

La troisième conséquence évoquée au cours de ce travail de recherche est la mécanisation des rapports, du fait des médias et des TICs. Même si elles saluent leur avènement, les populations de Baazra regrettent le remplacement du contact physique et des échanges par les médias, le téléphone et internet.

Pour eux, les médias sont à la base de la propagande des vices, des comportements déviants et de la déformation de l'éducation des jeunes.

"Aujourd'hui, les jeunes refusent d'aller travailler au champ. Ils s'adonnent à des pratiques déviantes, surtout quand ils sont ivres. Parfois, ils agressent des gens sous l'effet des stupéfiants. On peut donc dire que tout est gâté au village. Les jeunes ne sont plus sérieux. Et cela influence négativement l'économie villageoise » (chef du village Baazra I).

En somme, le développement visé par les ressortissants de Baazra est freiné par une dynamique communicationnelle marquée par la division et l'acculturation, du fait des nouvelles postures, du processus de prise de parole, de l'intervention non maitrisée et d'une forme inadaptée de démocratie.

\section{III.2. Discussion : La médiatisation d'une démocratie inadaptée et le rôle inapproprié de l'Etat}

Les médias ont joué un rôle important dans la promotion d'une culture démocratique inadaptée à la culture communicationnelle des gouro de Baazra. Les conflits émanant de ces changements sont préjudiciables au développement économique et social espéré par les membres de la communauté villageoise.

Dans un contexte de médiatisation accrue, le modèle communicationnel d'antan a changé à Baazra. La liberté d'expression a pris le pas sur le contrôle de l'information et la limitation des acteurs concernés par la prise de parole.

Pour les défenseurs de la liberté d'expression, cette nouvelle stratégie serait le symbole de l'évolution positive des idéologies. La liberté d'expression est un droit humain fondamental, pilier de toutes les libertés civiques. L'UNESCO, inscrite dans la logique des Nations Unies, a pour mandat spécifique de promouvoir la libre circulation des idées par le mot et par l'image. L'UNESCO vise à favoriser la mise en place de médias libres, indépendants et pluralistes sous formes imprimées, diffusées ou en ligne. Dans cette logique, le développement des médias encourage la liberté d'expression et contribue au renforcement de la paix, du développement durable, des droits de l'homme et de la lutte contre la pauvreté (UNESCO, 2019).

L'acte constitutif de l'organisation invite les États membres à travailler ensemble pour promouvoir la connaissance et la compréhension mutuelles des 
peuples à travers la libre circulation des idées. Pour l'UNESCO, la liberté d'expression est le fondement de toute démocratie.

Sur cette base, Baazra évolue vers un idéal type. Mais les résultats de cette étude montrent clairement que, ni la liberté d'expression, ni le système politique qui l'accompagne, ne sont bénéfiques à Baazra. Contrairement à ce que promeut l'UNESCO, cette situation détériore les relations familiales et encourage le non-respect du droit d'aînesse ou de la hiérarchie villageoise.

L'autre volet de la question qui se prête à débat est l'intervention de l'Etat et son système contraignant de désignation des chefs. Le chef traditionnel est défini par une double appartenance (Lombard, 1967). D’un côté, il symbolise la coutume, de l'autre, il représente l'administration.

Selon la Loi $\mathrm{n}^{\circ}$ 2014-451 du 05 août 2014 portant orientation de l'organisation générale de l'Administration Territoriale et les décrets subséquents, la chefferie traditionnelle reste dans un cadre structurel global. L'objectif visé est de rendre moderne et dynamique la gestion des villages, comme si le système traditionnel était statique. Cette politique est dite d'assimilation ou d'administration directe. Elle définit, selon Ouattara (2014) le contexte d'évolution du pouvoir politique «traditionnel ». Mais ladite intervention verticale, dans son application, n'a rien de démocratique, aux dires des ressortissants de Baazra qui contestent certains chefs. Si le développement et le progrès de l'éducation occidentale parviennent à Baazra par les médias et par l'autorité administrative, les conflits de chefferie sont la preuve que le processus adopté n'est pas en adéquation avec le champ particulier dudit village.

Dans cette logique, nous nous interrogeons avec Sylla (2006) sur l'existence ou non d'un modèle universel de la démocratie. Peut-on dire que le mode traditionnel de choix des dirigeants à Baazra n'est pas démocratique ? Si la démocratie est un pouvoir du peuple, alors elle existe dans les fondements traditionnels. Comme Sylla (ibid.), et en référence aux données de ce travail de recherche, l'on peut conclure qu'il n'existe pas de modèle universel de démocratie. Mais plus encore, le système traditionnel est naturellement dynamique, comme le soutient Ouattara (op. cit.). C'est aussi dans ce cadre que Bayart (1989, p.31) affirme que «Dans les faits, la tradition, non seulement n'était pas une, mais encore n'était ni immobile ni fermée. (...) Par ailleurs, les acteurs sociaux contemporains chevauchent sans arrêt les secteurs arbitrairement circonscrits de la tradition et de la modernité ».

L'étude révèle par ailleurs que la dynamique communicationnelle qui émerge de la liberté d'expression et de la démocratie occidentale médiatique en milieu rural est la cause d'instabilité et de conflits latents à Baazra. Désormais déterminée par d'autres valeurs que l'âge et la connaissance de la tradition, la prise de parole n'est plus réglementée. On assiste progressivement à une monétarisation des rapports sociaux et à la montée de l'individualisme. 
Dans ce contexte, la cohésion sociale est mise à mal et le développement économique et social involontairement freiné par un processus d'affirmation de soi, de sa raison et de la démonstration du tort des autres. A Baazra, chaque acteur symbolise une origine sociale, une famille, une prise de partie dans les conflits. Sur cette base, de nombreuses actions sont boycottées par une partie des acteurs.

\section{Conclusion Generale}

Communiquer en Afrique est un exercice difficile et protocolaire. Dans un pays comme la Côte d'Ivoire, qui sort d'une crise post-électorale et qui peine à se réconcilier, toutes les formes de communication sont importantes. Cette étude a permis de comprendre les implications sociales de la dynamique communicationnelle en milieu rural, plus précisément dans le village de Baazra. Il s'est agi d'apprécier les changements qui découlent du modèle communicationnel, en comparant les structures passées à celles du présent. Aussi, il a été question d'analyser les causes et les conséquences des modes actuels de communication. Le présent travail qui a adopté une approche qualitative, a mis en exergue l'impact des médias, de la démocratie à l'occidentale et l'émergence d'une liberté d'expression qui va à l'encontre des normes culturelles.

De fait, l'intervenant doit sa prise de parole à la médiatisation d'habitudes occidentales et à sa posture économique dans le contexte actuel. Mais les conflits récurrents à Baazra 1 et 2 traduisent la rupture entre cadre traditionnel et cadre moderne. Cela se perçoit à travers l'implication inappropriée de l'Etat. Dans ce registre, le développement et la stabilité communautaires sont remis en cause dans la désignation des chefs de villages. C'est pourquoi, l'étude s'achève par des suggestions émanant des enquêtés. La première envisage le recours à la tradition et à son institutionnalisation dans le processus de désignation des chefs de villages. A cet effet, il est souhaitable qu'à la base, les parents inculquent à leurs enfants les codes élémentaires de leur langue maternelle. A défaut d'un retour pur et simple aux valeurs africaines dans leurs originalités, comme deuxième suggestion, les enquêtés évoquent la contextualisation des valeurs démocratiques en matière de prise de parole dans les villages. La troisième suggestion qui ressort de l'étude, c'est l'instauration d'une médiation continue entre les organes du village et ceux de ses ressortissants vivant en zone urbaine. Il s'agit de la création d'un cadre permanent de communication entre eux. La non-interférence des idéologies politiques exogènes dans la gestion de la chefferie est citée comme quatrième suggestion issue de l'étude. Cette proposition vise à ne pas retarder le développement du village. Enfin, au regard du poids démographique des jeunes $(78,3 \%$ de la population de Baazra 1 et 2 ont moins de 35 ans, selon le recensement général de la population ivoirienne de 2014), leur 
responsabilisation est envisagée. L'ensemble de ces suggestions constitue des défis à relever en matière de dynamique communicationnelle pour le développement de Baazra et du milieu rural ivoirien en général. Pour les travaux futurs portant sur le même objet, il serait pertinent d'étudier les modalités de la cohabitation des modèles de communications traditionnels et modernes en vue du développement local.

\section{References :}

1. Abdallah-Pretceille, M. \& Porcher, L. (1996). Education et communication interculturelle. P.U.F.

2. Bayart, JF. (1989). L'État en Afrique : La politique du ventre. Fayard.

3. Commission Dialogue Vérité et Réconciliation (CDVR) (2014). Rapport final, République de Côte d'Ivoire.

4. République de Côte d'Ivoire (2014). Loi n 2014-451 du 05 août 2014 portant orientation de l'organisation générale de l'Administration Territoriale et les décrets subséquents. Direction générale de la décentralisation et du développement local.

5. Kajabika, V., Ghosn, C., \& Kiyindou, A. (2018). «Des nouvelles dynamiques médiatiques dans les zones périphériques », Communication, vol. 35/2. http://journals.openedition.org/communication/7792.

6. Kajabika, V. \& Kiyindou, A. (2018). «Introduction. "Réseaux sociaux numériques et nouvelles dynamiques médiatiques : des propositions aux contributions inédites" ",, French Journal for Media Research. Actes du colloque Médiatiques numériques. http:// frenchjournalformediaresearch.com/lodel/index.php?id=1594.

7. Lohisse, J. (1999). «L'anthropologie, la communication et leurs lieux », Recherches en communication, $\mathrm{n}^{\circ} 12,13-25$.

8. Lombard, J. (1967). Autorités traditionnelles et pouvoirs européens en Afrique Noire : Le déclin d'une aristocratie sous régime colonial, Presse de la fondation nationale des sciences politiques, Armand Colin.

9. République de Côte d'Ivoire (2014). Loi n ${ }^{\circ} 428$ du 14 juillet 2014 portant statut des Rois et Chefs Traditionnels de Côte d'Ivoire, $56^{\text {ème }}$ année, Journal Officiel de la République de Côte d'Ivoire, n¹1.

10. Ouattara, KA. (2014). « La chefferie africaine : Tradition, Modernité et Survivance », Revue ivoirienne d'histoire, n 23, 86-99.

11. Sylla, L. (2006). «Existe-t-il un modèle universel de démocratie ?», Comprendre..., $\mathrm{N}^{\circ} 3$. Les éditions CERAP.

12. UNESCO (2019). Favoriser la liberté d'expression. https://fr.unesco.org/themes/favoriser-libert\%C3\%A9-dexpression. 